\title{
Nanotechnology Building Blocks for Intervention with Alzheimer's Disease Pathology: Implications in Disease Modifying Strategies
} Nazem A ${ }^{1}$, Mansoori GA ${ }^{2 *}$

${ }^{1}$ Elmezzi Graduate School of Molecular Medicine, Feinstein Institute for Medical Research, Manhasset, NY, 11030, USA

${ }^{2}$ College of Engineering, University of Illinois at Chicago, 851 S. Morgan St.(M/C 063), Chicago, IL 60607-7052, USA

\section{Introduction}

Alzheimer's disease (AD) is the most common neurodegenerative disorder manifested by progressive loss of memory followed by irreversible dementia [1-3]. AD neurodegeneration is characterized by the loss of neurons preceded by cell membrane and cytoskeleton damage due to a complex of molecular pathways that finally lead to formation of amyloid- $\beta(A \beta)$ plaques and neurofibrillary tangles in the brain tissue [1-3]

Current treatments of $\mathrm{AD}$ can only partially alleviate symptoms, but there exists no cure to stop or slow neurodegeneration. The first major class of drugs used to treat $\mathrm{AD}$ was cholinesterase inhibitors which inhibit the proper functioning of the enzyme that breaks down acetylcholine. These drugs maintain a higher level of acetylcholine in the synaptic cleft thereby promote signaling and improve neuronal function; however, they do not prevent the underlying neurodegeneration [1-3]. On the other hand, all current diseasemodifying strategies targeting different molecular pathways involved in $\mathrm{AD}$ have failed in phase 3 clinical trials [4].

As reported in our recent detailed studies [2,3], major molecular pathways involved in $\mathrm{AD}$ can potentially be targeted through nanotechnology methods [5] and molecular building blocks (MBBs) [6] as it is shown in Figure 1. In summary, the current and envisioned applications of nanotechnology in neurology consist of neuroprotection, neuroregeneration and drug delivery beyond the blood brain barrier (BBB).

Among the above-mentioned applications, nanotechnology neuroprotective approaches can be of great implication in effective disease-modifying strategies for $\mathrm{AD}$, especially when the targeted molecular pathways are involved in more initial phases of $\mathrm{AD}$ pathogenesis. This report is focused on such neuroprotective approaches through nanotechnology molecular building blocks (MBBs). To this end, we first elaborate on molecular pathways of Alzheimer's disease targeted by each group of nanotechnology MBBs.

\section{Protection against Glutamate Excitotoxicity}

A major pathological event in AD is synapticfailure [9], characterized by excessive synaptic release of glutamate and/or impairment of glutamate clearance mechanisms in the synaptic cleft. Glutamate together with $\mathrm{D}$-serine are the primary excitatory neurotransmitters in the brain that activate voltage-dependent calcium channels through the N-methyl-D-aspartate (NMDA) receptors. Excessive synaptic glutamate, in turn, leads to a disruptive influx of calcium ions causing an overabundance of intracellular calcium, triggering several damaging pathways in neurons, and ultimately neuronal death (Figure 2) $[1,5,6]$. Therefore, a disease modifying approach against $\mathrm{AD}$ synaptic pathology could be preventing the excessive glutamate signaling by blocking the NMDA-receptor channels [5].

From the structural point of view, NMDA receptors are coassemblies of different receptor subunits from different subunit families: NR1 and NR2 as shown in Figure 3. Among these subunits, NR1 has the essential role in NMDA receptor function [8]. Co-assembly of different members of the above mentioned subunit families leads to composition of different NMDA receptor subtypes with discrepant biophysical and pharmacological properties [9]. A variety of binding sites for ligands exist in the structure of NMDA. These ligand/binding site interactions are almost subunit selective and result in regulation of NMDA receptor function [9] as shown in Figure 3.

The topologies of NMDA receptor subunits are almost the same. Each subunit consists of an extracellular N-terminal domain, four membrane (M 1-4) domains and an intracellular carboxyl terminal $[8,9]$. The extracellular domain (N-terminal) forms the glycosylated large part of each subunit, responsible for binding with ligands. Among agonist ligands, glycine binds to NR1 and glutamate binds to NR2 binding sites. The first and second intra-membranous domains are interconnected through an intramembrane loop. The M2 domain forms the receptor channel pore. The intracellular domain (carboxyl terminal) contributes to regulation of the receptor through second messenger system $[8,9]$.

As discussed before, if excessive NMDA signaling is not controlled, a high influx of calcium leads to the impairment of mitochondrial function, cellular energy failure and finally neurodegeneration [5]. Previously developed drugs which act as NMDA antagonists were discontinued due to blocking the normal NMDA signaling, a process that can also promote neurodegeneration [5]. Selective blockade of the excessive NMDA signaling is possible through optimization of NMDA receptor partial antagonism via nanotechnology molecular building blocks (MBBs) and their derivatives $[9,10]$.

A derivative of nanotechnology building blocks diamondoids, known as Memantine, shows effective partial NMDA receptor antagonism [6]. Memantine is a derivative of adamantine (1-amino3,5-dimethyladamantane) the smallest diamondoid molecule [10] (Figure 4). Diamondoids are cage-like saturated hydrocarbons and one of the promising nanotechnology MBBs for biological applications. Memantine acts as a low-affinity and noncompetitive receptor antagonist which unbinds following strong synaptic activation due to its voltage-dependency and fast unblocking kinetics. Therefore, Memantine prevents excessive glutamate from setting off too high a calcium influx, while still allowing normal NMDA receptor to function

${ }^{*}$ Corresponding author: Mansoori GA, Professor of Chemical and Bio Engineering \& Physics, Department of Bioengineering, University of Illinois at Chicago, USA; Tel: 1-312-996-5592; E-mail: mansoori@uic.edu

Received March 20, 2013; Accepted April 23, 2014; Published April 27, 2014

Citation: Nazem A, Mansoori GA (2014) Nanotechnology Building Blocks for Intervention with Alzheimer's Disease Pathology: Implications in Disease Modifying Strategies. J Bioanal Biomed 6: 009-014. doi:10.4172/1948-593X.1000101

Copyright: @ 2014 Nazem A, et al. This is an open-access article distributed under the terms of the Creative Commons Attribution License, which permits unrestricted use, distribution, and reproduction in any medium, provided the original author and source are credited. 


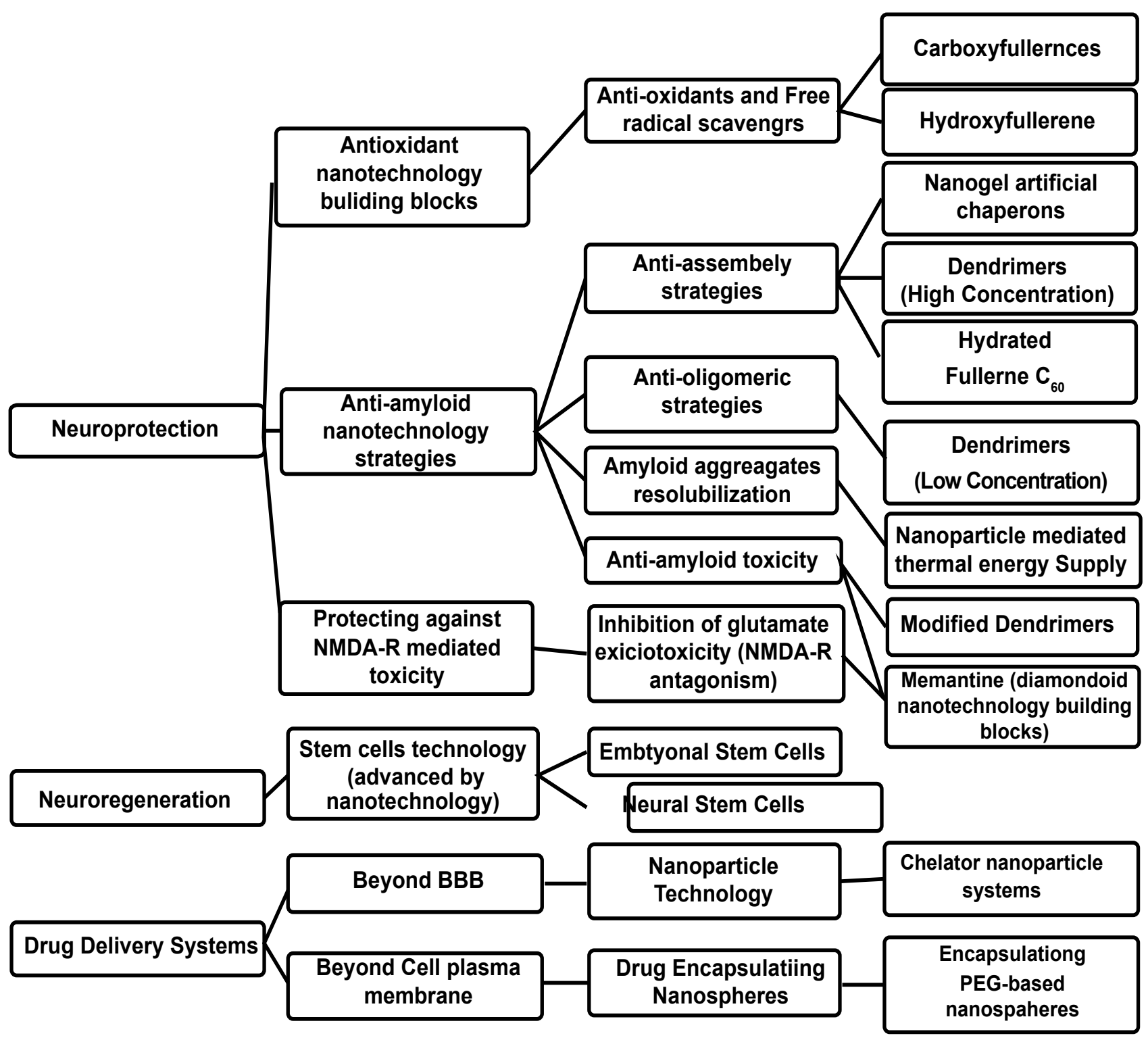

Figure 1: The summary of potential applications of nanotechnology in the treatment of Alzheimer's disease [2]

at normal concentrations of glutamate [5]. Memantine has become of great interest to researchers owing to such self-regulated mechanism that controls the amount of NMDA activation [11].

\section{Protection against Oxidative Stress}

There is compelling evidence on initial role of mitochondrial derived oxidative stress in $\mathrm{AD}$ pathogenesis [12]. Mitochondria are crucial organelles to the eukaryotic cells for both energy generation and regulation of programmed cellular death (apoptosis). During the cellular energy metabolism in the mitochondria, semi reduced oxygen species (ROS), including super oxide and hydrogen peroxide, are normally produced [13]. According to the AD mitochondrial cascade hypothesis [14], during aging, which is the main risk factor for sporadic $\mathrm{AD}$, the continual damage to mitochondrial DNA (mtDNA) leads to the malfunction of proteins of mitochondrial electron transfer chain
(ETC) [12-14]. The resultant decrease in ETC efficiency per se causes overproduction of ROS [13].

Oxidative stress happens when the production of ROS exceeds their neutralization. The ROS, unless neutralized, can contribute to some cytopathological reactions, including oxidation of mitochondrial DNA (mtDNA) and to a lesser degree nuclear DNA, as well as protein oxidation and lipid peroxidation. These pathological reactions, in turn, lead to cell death and degeneration [12-14]. It is noteworthy that, in addition to mitochondrial dysfunction, the interaction between toxic $\mathrm{A} \beta$ aggregates and cell membrane is another major source of oxidative stress in AD pathology.

Fullerene is one of the main nanotechnology building blocks with strong antioxidant and free radical scavenger properties, owing to its specific chemical structure that allows it to be linked (i.e. functionalized) 
(a) Physiological

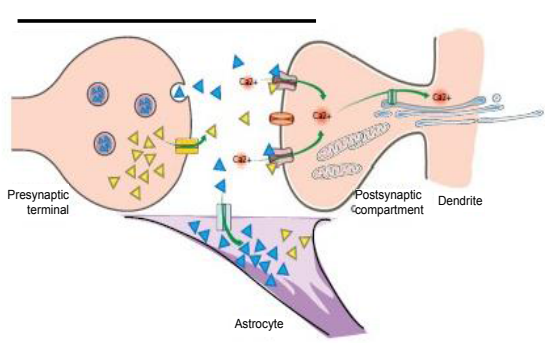

(b) Pathological

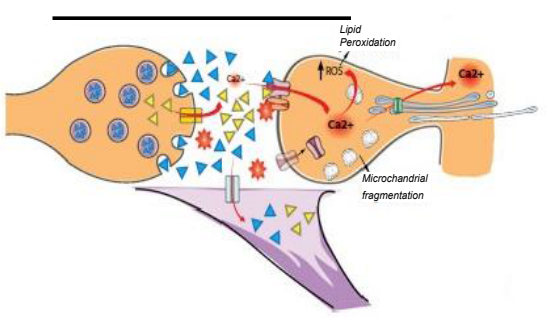

A $\beta$ Os

$\Delta$ Glutamate

D-serine

Mitochondria

Golgi complex

Synaptic

Vesicle

NMDAR

A $\beta O$ receptor

EAAt

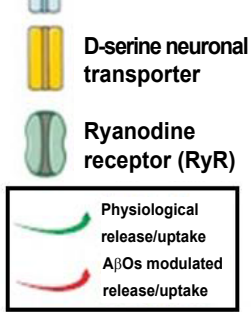

Figure 2: Schematic view of synaptic cleft in physiological and pathological conditions. In physiological condition, normal concentrations of glutamate and D-serine, the main excitatory amino acids, are essential for proper signal transfer between neurons. In pathological condition, $A \beta$ oligomers $(A \beta O)$ exert functional synaptic disruption and finally structural synaptic damage through a complex interaction between a putative $A B O$ receptors and NMDA receptors leading to synaptic failure. A key functional alteration is excessive glutamate release from presynaptic compartment and/or impaired glutamate clearance by excitatory amino acid transporters (EAAT) of astrocytes. Excessive glutamate over activates NMDA receptors, causing excitotoxicity through pathological influx of $\mathrm{Ca} 2+$. Excessive intracellular $\mathrm{Ca} 2+$ subsequently triggers various pathological events such as membrane lipid peroxidation, formation of reactive oxygen species, mitochondrial fragmentation and ultimately cell death [5].

to several active chemical groups in a 3-dimensional orientation [1517]. Fullerene molecule is composed of a three-dimensional array of carbon atoms, evenly spaced in a pattern similar to rhombuses on a soccer ball [15-17].

In vitro studies on Fullerenol, a water-soluble hydroxylfunctionalized derivative of fullerene, have shown neuroprotective properties against $A \beta 42$ via both antioxidant reactions and inhibition of $\mathrm{A} \beta 42$-induced $\mathrm{Ca}^{2+}$ neurotoxicity [17]. Likewise, carboxy fullerenes (malonic acid derivative of $\mathrm{C}_{60},\left\{\mathrm{C}_{63}\left[(\mathrm{COOH})_{2}\right]_{3}\right\}$ ) protect cultured cortical neurons from $\mathrm{A} \beta 42$ induced oxidative stress and neurotoxicity [18]. Interestingly, the application of carboxy fullerenes blocked the A 342 induced neuronal apoptotic death as well [18].

In addition, hydrated $\mathrm{C}_{60}$ fullerene $\left(\mathrm{C}_{60} \mathrm{HyFn}\right)$ is shown to protect neurons against $\mathrm{A} \beta$ induced neurodegeneration in vivo, presumably owing to both its anti-oxidant and anti-amyloid properties [19]. The molecular mechanism of $\mathrm{C}_{60} \mathrm{HyFn}$ anti-amyloid properties is not clear yet [19]. Anti-amyloid properties were also attributed to sodium fullerenolate $\mathrm{Na} 4\left[\mathrm{C}_{60}(\mathrm{OH}) \sim 30\right](\mathrm{NaFL})$, a water soluble polyhydroxylated fullerene derivative [20]. NaFL destroys mature amyloid fibrils and at the same time prevents from $A \beta$ aggregation and fibril formation. Thus, increased concentration of $A \beta$ oligomers due to $A \beta$ fibril break-down might be a source of potential toxicity in vivo. Of course, in vitro studies reported NaFL toxicity is exceptionally low. However, this is to be confirmed in pre-clinical in vivo trials[20].

\section{Protection against Amyloid- $\beta$ induced Synaptic and Neuronal Damage}

In early stages of $\mathrm{AD}$, symptoms are more due to Amyloid- $\beta(\mathrm{A} \beta)$ damage to synapses rather than its damage to neurons themselves. For example, the early memory impairment is more related to $A \beta$ synaptotoxicity than its neurotoxicity [21]. Therefore, the synaptic degeneration and its presentations in early clinical symptoms of $\mathrm{AD}$ precede neuronal degeneration and death $[22,23]$.

Growing evidence suggests the culprit molecules in synaptic

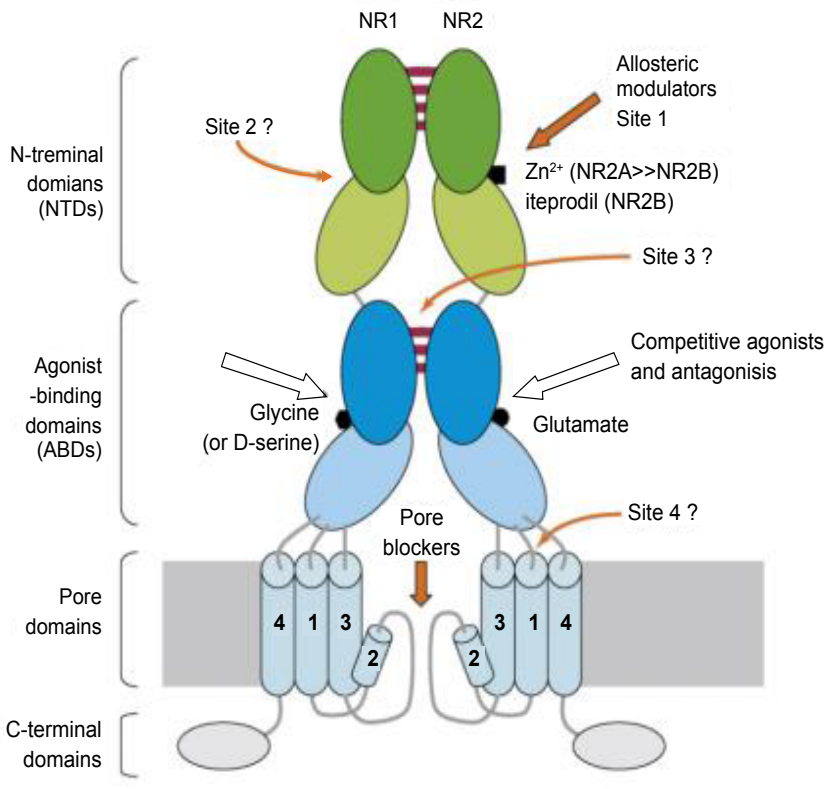

Figure 3: Schematic representation of NMDA receptor and its potential binding sites for ligands [10]: As it is shown most of NMDA receptor subtypes have quaternary structure, composing of two NR1, and two NR2 subunits (here only one pair of subunits is schemed). The large extracellular domain consists of two regions: NTD and ABD. The ABD region contains the site for binding with ligands. NR1 allows binding with glycine (or D-serine) and glutamate occupies the NR2 binding site. Other agonist and antagonist ligands could bind at the same sites (as pointed with large arrows). The thick orange arrows show the site for binding with allosteric modulators (like endogenous zinc) and the thin orange arrows indicates the putative sites for both positive and negative allosteric modulators. The ion channel domain also provides binding sites for pore blockers such as endogenous $\mathrm{Mg}++$, Ketamine and Memantine, all acting as uncompetitive antagonists.

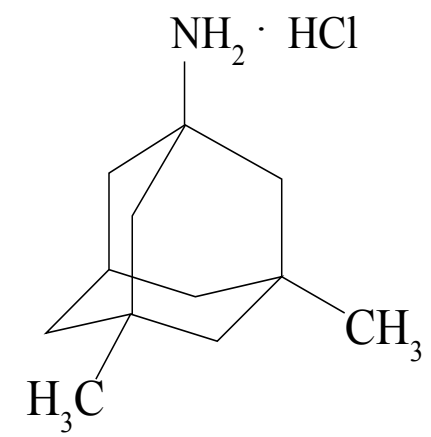

Figure 4: Molecular structure of NAMENDA (Memantine. $\mathrm{HCl}$ ). NAMENDA is the commercial medicine name for the neutralized Memantine [6] 

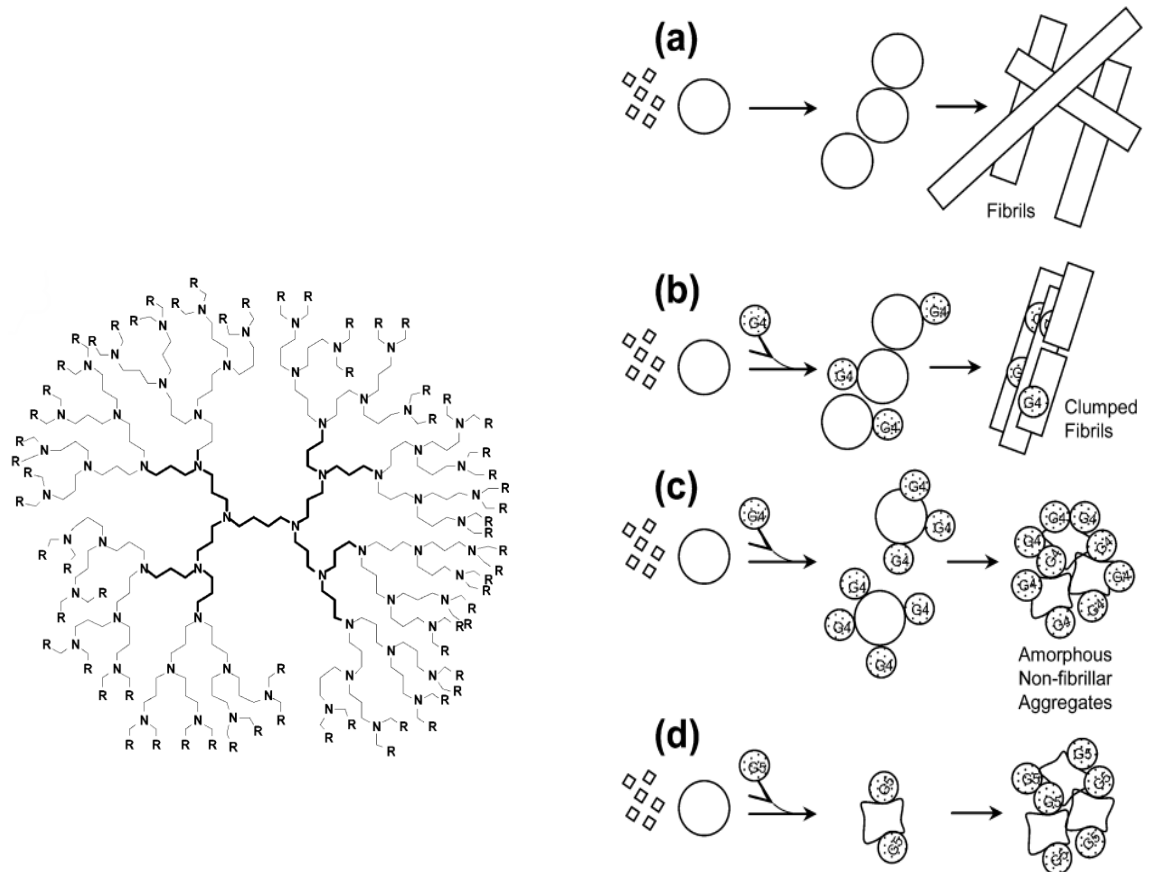

Figure 5 Structure and function of PPI- Mal dendrimers. (a) Chemical structure of PPI-G4-Mal dendrimer. (b) Schematic representation of different dendrimer-amyloid interactions, and morphology of the resulting aggregated species (a) Polymerization process of $A \beta(1-40)$ : monomeric peptide $(\diamond)$ assembles as peptide oligomers (O); peptide oligomers combine into prefibrillar structure (OOO) and eventually convert into fibrils ( $\square \square$ ). (b) At low dendrimer-peptide ratios, the interaction of the PPI-G4-Mal dendrimer with the monomeric peptide does not prevent the fibrillization, but clumps fibrils (c) At high dendrimer-peptide ratios, PPI-G4-Mal prevents the fibrillization, leading to the formation of dendrimer-peptide oligomer complexes. These complexes combine in the form of granular nonfibrillar amorphous aggregates. (d) PPI-G5-Mal changes the peptide oligomeric structures, probably through formation of a higher number of hydrogen bonds per dendrimer (even at low dendrimer-peptide ratios), thus preventing the fibrillization and leading to the formation of amorphous aggregates [36].

degeneration are the soluble oligomeric species of $A \beta(A \beta O)$, and the process is independent of the levels of amyloid precursor protein (APP) expression, $\mathrm{A} \beta$ monomers and amyloid depositions [22,23]. The exact downstream mechanisms of $A \beta O$ on synaptic degeneration are not clear yet. However, postsynaptic terminals are shown to be more affected than pre-synaptic terminals in $\mathrm{A} \beta \mathrm{O}$ induced toxicity (Figure 2) [5]. Interestingly, $A \beta O$ induced synaptic disruptions are more functional in the beginning, and are followed by structural disruptions later in the disease process. The functional synaptic impairment mainly include a reversible disruption in long term potentiation [24,25].

Some signaling pathways have been suggested to account for the functional toxic effects of $\mathrm{A} \beta \mathrm{O}$. The backbone of these pathways is a putative synaptic terminal receptor with high affinity to $\mathrm{A} \beta \mathrm{O}$, which is not yet fully characterized $[5,25]$. However, there is strong supporting evidence regarding association of $\mathrm{A} \beta \mathrm{O}$ with some synaptic terminal receptors, including Fyn, Arc, glutamate receptors and PSD 95 protein. It is hypothesized that specific interactions of $A \beta O$ with $\mathrm{PSD}-95$ protein lead to a displacement of Fyn, which is tethered by PSD-95 protein to the signaling chain [26]. Displacement of Fyn receptor will then inhibit the c-AMP response element-binding (CREB) protein signaling ending in synaptic dysfunction and eventually neuronal death [26].

Not only the extracellular $\mathrm{A} \beta \mathrm{O}$, but also the intra-membranous and intracellular $\mathrm{A} \beta \mathrm{O}$ can have toxic effects on synaptic terminals. We have discussed the intra-membranous oligomerization of $A \beta$ and its subsequent permeabilization effect on the plasma membrane as a neurotoxic mechanism elsewhere $[2,3]$. In addition to the same intra-membranous oligomerization mechanism, intracellular $\mathrm{A} \beta$ oligomerization is proposed as a mechanism for synaptic degeneration following intra terminal accumulation of $A \beta$ [27].

A $\beta$-synaptotoxicity may also occur through localized apoptotic changes as evidenced by caspase activation in neurites [28]. Caspase is the name for a family of cysteine proteases that are responsible for regulating apoptosis in mammalian cells. However, it is not completely proven whether the activation of caspases in $\mathrm{AD}$ causes classic apoptotic effects or if it merely performs some apoptosis-associated molecular dysfunctions in the neurons [28]. Some caspases, especially caspase-6, have been recently suggested as the first molecular responses of the insulted neurons and as triggers for further disruptions in the structure of neuronal cytoskeleton and subsequently in the neuronal trafficking $[28,29]$.

Blocking toxicity of $\mathrm{A} \beta \mathrm{O}$ has been widely studied as a disease modifying strategy for AD. To date, dendrimers are one of most validated nanotechnology designed structures with anti-amyloid properties [34]. Dendrimers are highly branched macromolecular structures with globular shape (Figure 5) and a densely packed surface [30-34]. Beside their potent anti-amyloid effects, however, most dendrimeric structures exert cytotoxic effects on neurons. Therefore, designing dendrimers with minimal toxicity has been the focus of several recent studies. Recently, minimally toxic dendrimers like poly- $\mathrm{L}$ lysine dendrimers D3 and D5 were found with strong anti-amyloid properties as demonstrated in human neuroblastoma SH-SY5Y cells. In addition, $\mathrm{D} 3$ lysine dendrimers, attenuated $\mathrm{A} \beta$ induced increases in $\mathrm{K}^{+}$ channel current [35]. Interestingly, following intraventricular injection 
of fluorescence-labelled D3 dendrimers to rat brain, dendrimers were found distributed to both parietal cortex and hippocampus, major regions involved in $\mathrm{AD}$, without any sign of cell death throughout the brain [35].

Similarly, maltose (Mal) decorated glycodendrimers, poly (propyleneimine) (PPI) dendrimers have also shown minimal toxicity in vitro [36]. Among them, specifically fourth (PPI-G4-Mal) and fifth (PPI-G5-Mal) generation glycodendrimers interfere with amyloid peptide $A \beta$ (1-40) fibrillization in a generation dependent manner: PPI-G5-Mal blocks amyloid fibril formation and PPI-G4-Mal clumps fibrils together (Figure 5) [36]. Blockade of amyloid fibril formation through PPI-G5-Mal leads to generation of granular non-fibrillar amorphous aggregates (Figure 5B). Likewise, PPI-G4-Mal fibril clumping leads to amorphous aggregates at high dendrimer-peptide ratios, but clumped fibrils at low ratios (Figure 5B). Fibril clumping reduces amyloid toxicity whereas amorphous aggregates are toxic to cells [36]. Of course, no cellular toxicity was found due to PPI-G4-Mal or PPI-G5-Mal themselves [36]. Further studies on in vivo effects of glycodendrimers are required to test their biocompatibility as antiamyloidogenic compounds for $\mathrm{AD}[36]$.

Interestingly, in a recent study, cationic phosphorus-containing dendrimers (CPD) (generations 3 and 4) were proved capable of interfering with both $\mathrm{A} \beta$ and MAP-Tau aggregation processes [37]. In this study, the fragment of amyloid peptide $\left(\mathrm{A} \beta_{1-28}\right)$, which is the core region responsible for aggregation, was used as a model for full-length $\mathrm{A} \beta$ monomer [37]. The cytotoxicity associated with formed fibrils and intermediate products of $A \beta_{1-28}$ aggregation decreased in the presence of CPDs via regulation of the fibrillization process [37].

Generally, dendrimers regulate $A \beta$ fibrillization in a concentration dependent manner. In low concentrations, dendrimers facilitate $A \beta$ fibrillization, whereas in higher concentrations, they block $A \beta$ fibrillization $[33,34]$, either via their binding with peptide monomers or through blocking the end of protofibrils and fibrils [34]. The therapeutic importance of this dualistic behavior of dendrimers is rooted in the recently described protective, rather than toxic role of $A \beta$ fibrillization. Based on this paradigm, $A \beta$ fibrillization is a natural mechanism to neutralize the toxic $\mathrm{A} \beta \mathrm{O}$, which can be considered as an alternative way to compensate for the impaired $A \beta$ clearance mechanisms in $\mathrm{AD}$ brain. In this regard, lower concentration of dendrimers could contribute to disease modification of $\mathrm{AD}$ through neutralizing toxic $A \beta O$ s via enhancement of $A \beta$ fibrillization [33,34]. An exception may be gallic acid-triethylene glycol dendrimer decorated with 27 terminal morpholine groups ([G3]-Mor) that facilitate amyloid fibirilization in high concentrations [38]. Interestingly, in line with the abovementioned notion, the results of G3-Mor and $A \beta$ interaction showed concomitant increase in $A \beta$ fibril formation and decrease in $\mathrm{A} \beta$-induced toxicity [38].

\section{Conclusions}

Thus far, all disease modifying strategies designed to slow or halt the Alzheimer's disease molecular pathogensis have failed in phase-3 clinical trials [4]. Future perspective on finding a cure for AD is not bright, without any considerable shift in the paradigms explaining the underlying mechanisms of $\mathrm{AD}$, establishing and validating relevant animal models used in preclinical studies [39] and methodologies used in drug design and discovery. While toxic side effects are still the major concern about different nanoparticles, biochemical features of these particles show potentials of a methodological breakthrough in targeted drug delivery to specific molecules involved in $\mathrm{AD}$ pathogenesis.

Recently, disease modifying potentials have been suggested with Memantine [40,41], a derivative of adamantane the smallest diamondoid molecule [42,43]. Diamondoids are key molecular building blocks for nanotechnology with several biological implications [44], including NMDA receptor antagonism as shown in NAMENDA. Clinically, NAMENDA is beneficial for treatment of moderate to severe AD.

As the currently dominant paradigm of $\mathrm{AD}$ pathogenesis emphasizes on $A \beta$ induced toxicity, several nanotechnology building blocks have shown neuroprotective effects against different mechanisms through which $A \beta$ damages neurons. For instance, $A \beta$ damage to synapses and neuronal cell membrane could be restrained via anti-amyloid (e.g. dendrimers) and anti-oxidant (e.g. fullerenes) nanotechnology building blocks respectively.

It is noteworthy that beside efforts towards effective disease modifying agents for $\mathrm{AD}$, nanotechnology research is also focusing on drug delivery systems that can allow targeted delivery of both current symptomatic and envisioned disease-modifying agents to the brain $[2,45]$, thus providing higher efficacy with minimal toxicity.

In conclusion, nanotechnology can provide potent agents to modify the course of certain pathological mechanisms involved in AD. However, translation of these strategies to actual modification of Alzheimer's disease course in human subjects lies largely not only on biocompatibility of these agents, but also on the accuracy of our current paradigms about molecular mechanisms underlying AD [46].

\section{References}

1. Sonkusare SK, Kaul CL, Ramarao P (2005) Dementia of Alzheimer's disease and other neurodegenerative disorders--memantine, a new hope. Pharmacol Res 51: 1-17.

2. Nazem A, Mansoori GA (2008) Nanotechnology solutions for Alzheimer's disease: advances in research tools, diagnostic methods and therapeutic agents. J Alzheimers Dis 13: 199-223.

3. Nazem A, Mansoori GA (2011) Nanotechnology for Alzheimer's disease detection and treatment. Insciences J 1: 169-193.

4. Mangialasche F, Solomon A, Winblad B, Mecocci P, Kivipelto M (2010) Alzheimer's disease: clinical trials and drug development. Lancet Neurol 9: 702-716.

5. Paula-Lima AC, Brito-Moreira J, Ferreira ST (2013) Deregulation of excitatory neurotransmission underlying synapse failure in Alzheimer's disease. Neurochem 126: 191-202.

6. Parsons CG, Danysz W, Quack G (1999) Memantine is a clinically well tolerated $\mathrm{N}$-methyl-D-aspartate (NMDA) receptor antagonist--a review of preclinical data. Neuropharmacology 38: 735-767.

7. Pomponi M, Bria P, Pomponi M (2008) Is Alzheimer's disease a synaptic disorder? J Alzheimers Dis 13: 39-47.

8. Gardoni F, Di Luca M (2006) New targets for pharmacological intervention in the glutamatergic synapse. Eur J Pharmacol 545: 2-10.

9. Paoletti P, Neyton J (2007) NMDA receptor subunits: function and pharmacology. Curr Opin Pharmacol 7: 39-47.

10. Mansoori GA, Arauio PLB, Arauio ES (2012)Diamondoid Molecules: With Applications in Biomedicine, Materials Science, Nanotechnology\& Petroleum Science.

11. Volbracht C, van Beek J, Zhu C, Blomgren K, Leist M (2006) Neuroprotective properties of memantine in different in vitro and in vivo models of excitotoxicity. Eur J Neurosci 23: 2611-2622.

12. Nunomura A, Perry G, Aliev G, Hirai K, Takeda A, et al. (2001) Oxidative damage is the earliest event in Alzheimer disease. J Neuropathol Exp Neurol 60: 759-767.

13. Mancuso M, Coppede F, Migliore L, Siciliano G, Murri L (2006) Mitochondrial 
Citation: Nazem A, Mansoori GA (2014) Nanotechnology Building Blocks for Intervention with Alzheimer's Disease Pathology: Implications in Disease Modifying Strategies. J Bioanal Biomed 6: 009-014. doi:10.4172/1948-593X.1000101

dysfunction, oxidative stress and neurodegeneration. J Alzheimers Dis 10: 5973.

14. Swerdlow RH, Khan SM (2004) A "mitochondrial cascade hypothesis" for sporadic Alzheimer's disease. Med Hypotheses 63: 8-20.

15. Vakili-Nezhaad GR, Mansoori GA, Ashrafi AR (2007) Symmetry Property of Fullerenes. J Computl Theor Nanoscience 4: 1202-1205.

16. Dugan LL, Gabrielsen JK, Yu SP, Lin TS, Choi DW (1996) Buckminsterfullerenol free radical scavengers reduce excitotoxic and apoptotic death of cultured cortical neurons. Neurobiol Dis 3: 129-135.

17. Huang HM, Ou HC, Hsieh SJ, Chiang LY (2000) Blockage of amyloid beta peptide-induced cytosolic free calcium by fullerenol-1, carboxylate $\mathrm{C} 60$ in PC12 cells. Life Sci 66: 1525-1533.

18. Dugan LL, Turetsky DM, Du C, Lobner D, Wheeler M, et al. (1997) Carboxyfullerenes as neuroprotective agents. Proc Natl AcadSci USA 94: 9434-9439.

19. Makarova EG, Gordon RY, Podolski IY (2012) Fullerene C60 prevents neurotoxicity induced by intrahippocampal microinjection of amyloid-beta peptide. J NanosciNanotechnol 12: 119-126.

20. Bobylev AG, Kornev AB, Bobyleva LG, Shpagina MD, Fadeeva IS, et al. (2011) Fullerenolates: metallatedpolyhydroxylated fullerenes with potent anti-amyloid activity. Org Biomol Chem 9: 5714-5719.

21. Rowan MJ, Klyubin I, Wang Q, Hu NW, Anwyl R (2007) Synaptic memory mechanisms: Alzheimer's disease amyloid beta-peptide-induced dysfunction. Biochem Soc Trans 35: 1219-1223.

22. Scheff SW, Price DA (2006) Alzheimer's disease-related alterations in synaptic density: neocortex and hippocampus. J Alzheimers Dis 9: 101-115.

23. Walsh DM, Selkoe DJ (2004) Deciphering the molecular basis of memory failure in Alzheimer's disease. Neuron 44: 181-193.

24. Lee HG, Moreira PI, Zhu X, Smith MA, Perry G (2004) Staying connected: synapses in Alzheimer disease. Am J Pathol 165: 1461-1464.

25. Gong Y, Chang L, Viola KL, Lacor PN, Lambert MP, et al. (2003) Alzheimer's disease-affected brain: presence of oligomeric A beta ligands (ADDLs) suggests a molecular basis for reversible memory loss. Proc Natl Acad Sci U S A 100: 10417-10422.

26. Gylys KH, Fein JA, Yang F, Wiley DJ, Miller CA, et al. (2004) Synaptic changes in Alzheimer's disease: increased amyloid-beta and gliosis in surviving terminals is accompanied by decreased PSD-95 fluorescence. Am J Pathol 165: 1809-1817.

27. NazemAMansoori GA (2009) The role of molecular interactions in the pathogenesis of Alzheimer's disease: could nanotechnology put a stop to it? Journal of Cerebral Blood Flow \& Metabolism 29: S273-S287

28. Roth KA (2001) Caspases, apoptosis, and Alzheimer disease: causation, correlation, and confusion. J Neuropathol Exp Neurol 60: 829-838.

29. LeBlanc AC (2005) The role of apoptotic pathways in Alzheimer's disease neurodegeneration and cell death. Curr Alzheimer Res 2: 389-402.

30. Bosman AW1, Janssen HM, Meijer EW (1999) About Dendrimers: Structure Physical Properties, and Applications. Chem Rev 99: 1665-1688.

31. Nikakhtar A, Nasehzadeh A, Mansoori GA (2007) Formation and Stability Conditions of DNA-Dendrimer Nano-Clusters. J Comput \& Theor Nanoscience 4: 521-528.

32. Nikakhtar A, Nasehzadeh A, Naghibi H and Mansoori GA (2005) DNAdendrimernano-cluster electrostatics prediction with the non-linear PoissionBoltzman equation. J. Comput \& Theor Nanoscience 2: 378-384.

33. Klajnert B, Cortijo-Arellano M, Cladera J, Bryszewska M (2006) Influence of dendrimer's structure on its activity against amyloid fibril formation. Biochem Biophys Res Commun 345: 21-28.
34. Klajnert B, Cortijo-Arellano M, Bryszewska M, Cladera J (2006) Influence of heparin and dendrimers on the aggregation of two amyloid peptides related to Alzheimer's and prion diseases. Biochem Biophys Res Commun 339: 577-582.

35. Neelov IM, Janaszewska A, Klajnert B, Bryszewska M, Makova NZ, et al. (2013) Molecular properties of lysine dendrimers and their interactions with $A \hat{l}^{2}$ peptides and neuronal cells. Curr Med Chem 20: 134-143.

36. Klementieva O, Benseny CN, Gella A, Appelhans D, Voit B, et al. (2011) Dense shell glycodendrimers as potential nontoxic anti-amyloidogenic agents in Alzheimer's disease. Amyloid-dendrimer aggregates morphology and cell toxicity. Biomacromolecules 14: 3903-3909.

37. sWasiak T, lonov M, Nieznanski K, Nieznanska H, Klementieva O, et al. (2012) Phosphorus dendrimers affect Alzheimer's $(\mathrm{A} \beta 1-28)$ peptide and MAP-Tau protein aggregation. Mol Pharm 9: 458-469.

38. Klajnert B, Wasiak T, Ionov M, Fernandez VM, Sousa HA, et al. (2012) Dendrimers reduce toxicity of $A \beta$ 1-28 peptide during aggregation and accelerate fibril formation. Nanomedicine 8: 1372-1378.

39. Nazem A, Sankowski R, Bacher M, Al-Abed Y (2014) Rodent models of neuroinflammation for Alzheimer's disease.

40. Reisberg B, Doody R, Stöffler A, Schmitt F, Ferris S, et al. (2003) Memantine in moderate-to-severe Alzheimer's disease. N Engl J Med 348: 1333-1341.

41. Lipton SA (2004) Paradigm shift in NMDA receptor antagonist drug development: molecular mechanism of uncompetitive inhibition by memantine in the treatment of Alzheimer's disease and other neurologic disorders. J Alzheimers Dis 6: S61-74.

42. Mansoori GA (2007) Diamondoid Molecules. Advances in Chemical Physics 136: $207-258$.

43. Mansoori GA, Mohazzabi P, McCormack P, Jabbari S (2007) Nanotechnology in cancer prevention, detection and treatment: bright future lies ahead. WRSTSD 4: 226-257.

44. Ramezani $\mathrm{H}$ and Mansoori GA (2007) Diamondoids as Molecular Building Blocks for Nanotechnology. In Molecular Building Blocks, Springer, New York USA.

45. Di Stefano A, lannitelli A, Laserra S, Sozio P (2011) Drug delivery strategies for Alzheimer's disease treatment. Expert Opin Drug Deliv 8: 581-603.

46. Tabaton M, Perry G, Zhu X, Lee HG, Casadesus G, et al. (2010) Memantine: "hypothesis testing" not "disease modifying" in Alzheimer's disease. Am J Pathol 176: 540-541. 